TERRA. Revista de Desarrollo Local e-ISSN: 2386-9968

Número 9 (2021), 195-202

DOI 10.7203/terra.9.22900

IIDL - Instituto Interuniversitario de Desarrollo Local

\title{
Ejemplo de custodia del territorio en Teruel (España): el caso de Mas Blanco y Recartografías
}

\author{
María Sánchez-Murciano \\ Graduada en Ciencias Ambientales, Universidad de Valencia (España). Recartografías. \\ maria.s.murciano@gmail.com
}

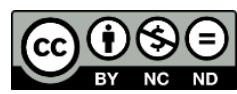

Esta obra se distribuye con la licencia Creative Commons Reconocimiento-NoComercial-SinObraDerivada 4.0 Internacional 


\title{
SECCIÓN EXPERIENCIAS Y BUENAS PRÁCTICAS
}

\author{
Ejemplo de custodia del territorio en Teruel (España): el caso de Mas Blanco y Recartografías
}

Resumen: Vivimos rodeados de elementos que naturales y antrópicos que tienen un valor por sí mismos, y que merecen un cuidado y protección para mantenerse a lo largo del tiempo, a salvo de una posible degradación. La custodia del territorio aparece como una herramienta más en este sentido, si bien con el añadido de permitir la gestión privada. Esto significa que, mediante su uso, la sociedad puede llegar a espacios o elementos donde la administración pública no lo hace. La formalización de un acuerdo de custodia del territorio puede ser verbal o escrito, y tiene lugar entre el propietario de un inmueble, elemento natural o territorio, y la entidad de custodia que se ofrece a gestionarlo. En España encontramos distintas figuras de organización de estos acuerdos. La custodia del territorio se aplica generalmente sobre espacios naturales, de titularidad pública o privada. Y en este ámbito trabaja Recartografías, que se presenta como una asociación cultural sin ánimo de lucro que trabaja mediante contratos de custodia con el ayuntamiento de San Agustín (Teruel), gracias al cual se les cedió durante siete años los edificios comunales de Mas Blanco, masada perteneciente a dicho municipio. El proyecto tenía un doble objetivo: por un lado, evitar la pérdida de los edificios de la masada, destacando entre ellos la escuela; y, por otro lado, recuperar los aprovechamientos comunitarios y otras actividades que tenían lugar, como la agricultura, cada vez más minoritarias. Sin embargo, son pocas las ayudas destinadas a este tipo de proyectos o entidades. Hay subvenciones, sobre todo autonómicas, estatales, y cada vez más, europeas, pero para poder redactar un proyecto aspirante a estas ayudas es necesario un equipo de gente comprometida con la asociación, y este es el segundo hándicap: la falta de recursos humanos. Pero la experiencia de Recartografías nos enseña que es posible llevar a cabo proyectos de recuperación de la memoria histórica a base de constancia y compromiso.

Palabras clave: espacios rurales, desarrollo territorial, comunidad, cohesión social, compromiso, Mas Blanco (San Agustín).

Recibido: 28 de diciembre de 2021

Devuelto para revisión: -

Aceptado: 28 de diciembre de 2021

\section{Referencia / Citation:}

Sánchez-Murciano, M. (2021). Ejemplo de custodia del territorio en Teruel (España): el caso de Mas Blanco y Recartografías. TERRA. Revista de Desarrollo Local, (9), 195-202. DOI 10.7203/terra.9.22900 


\section{INTRODUCCIÓN: ¿QUÉ ES LA CUSTODIA DEL TERRITORIO?}

El término "custodia" proviene del latín "custodiae", y responde al verbo custodiar, que según la Real Academia de la Lengua Española significa "guardar algo con cuidado y vigilancia". Custodiar significa cuidar.

Basora y Sabaté (2006) definen la custodia del territorio como "una estrategia más para conservar los valores naturales, culturales y paisajísticos de una zona determinada" ( $p$. 10). Vivimos rodeados de elementos que naturales y antrópicos que tienen un valor por sí mismos, y que merecen un cuidado y protección para mantenerse a lo largo del tiempo, a salvo de una posible degradación. Ya existen distintas herramientas para ello, figuras de protección municipales, autonómicas o nacionales, por ejemplo, y entre las que la custodia del territorio es una más. La diferencia principal es que puede ser un instrumento de gestión privada. Esto significa que, mediante su uso, la sociedad puede llegar a espacios o elementos donde la administración pública no lo hace. Es por tanto una iniciativa no sólo privada, sino que tiene su origen en las bases de la sociedad: son las personas, mediante asociaciones o movimientos sociales, quienes buscan organizarse para proteger aquellos elementos que consideran de importancia y que sienten desprotegidos o ante un riesgo inminente de degradación.

La formalización de un acuerdo de custodia del territorio puede ser verbal o escrito, y tiene lugar entre el propietario de un inmueble, elemento natural o territorio, y la entidad de custodia que se ofrece a gestionarlo. Una entidad de custodia no debe necesariamente ser una entidad privada. Una administración o institución pública también puede hacer uso de esta herramienta para la gestión de un bien privado.

El origen de la custodia del territorio lo encontramos en Estados Unidos a finales del s. XIX (Ruiz et al., 2018), en un contexto social muy definido: por un lado, el rechazo de la sociedad estadounidense a las experiencias en gestión existentes por parte del gobierno y las administraciones públicas; $y$, por otro lado, la necesidad de volver a reorganizar un país fuertemente afectado por un conflicto bélico reciente (Moreno, 2017). Posteriormente, este instrumento se extendió a través de los continentes europeos y americanos. En el caso de España, esta herramienta se formaliza en la "Declaración de Montesquiu", en el año 2000, promovida por instituciones y entidades de las Islas Baleares y Cataluña (Villagrasa, 2016).

En España encontramos distintas figuras de organización de estos acuerdos. Así, por un lado, tenemos las entidades de custodia, aquellas que formalizan el acuerdo y gestionan el elemento o territorio acordado. Y, por otro lado encontramos las redes de custodia, plataformas autonómicas en las cuales se engloban estas entidades. Y a nivel estatal encontramos la "Plataforma Nacional de Custodia del Territorio", que aglutina a todas ellas, y organiza congresos anuales que pretenden servir de espacio para intercambiar información y experiencias, así como para presentar los distintos proyectos y resultados obtenidos. Esta plataforma además ha elaborado un "mapa de custodia", en el cual se geolocalizan todos los lugares, a nivel nacional, en los que se ha firmado un acuerdo de custodia, y brinda una breve información sobre el mismo. El mapa está abierto a consulta pública, y puede encontrarse sin problema en Internet. En total, en 2021, se encuentran registrados 2.197 proyectos en custodia (Plataforma de Custodia del Territorio).

La custodia del territorio se aplica generalmente sobre espacios naturales, de titularidad pública o privada. Pueden ser desde campo de cultivos, parcelas acotadas sin ningún tipo de aprovechamiento, parcelas o espacios forestales, incluso tramos fluviales. También 
pueden aplicarse sobre elementos concretos, como por ejemplo un árbol singular o un monumento natural, o también sobre elementos de origen antrópico. Por ejemplo, una casa. O un conjunto de casas.

De las experiencias de custodia de espacios "terrestres", nacen dos nuevas iniciativas: la custodia marina y la custodia fluvial. En este caso, la custodia se centra en proteger y gestionar áreas incluidas en ecosistemas marinos y fluviales. A diferencia de las masas de agua estancadas (lagos y humedales), las masas de agua marinas y acuáticas no estancadas pertenecen al dominio público hidráulico o marítimo terrestre, de manera que el agente responsable de su gestión es la administración pública competente.

Los objetivos son los mismos que la custodia del territorio: organizaciones sociales que pretenden llegar donde no lo hace la administración pública. Así, mediante contratos de custodia, se cede la gestión de estos espacios, para la realización de proyectos e iniciativas de gestión, conservación y restauración de estos ecosistemas.

Un ejemplo de custodia marina es el liderado por la ONG SUBMON, que coordina la red catalana de custodia del territorio (Xarxa de Custòdia del Territori) y trabaja dese 2009 impulsando acuerdos de custodia marina en esta comunidad autónoma, en zonas litorales que se encuentran bajo distintas figuras de protección (Plataforma de Custodia del Territorio).

Por otro lado, encontramos un ejemplo de custodia fluvial en la Fundación LIMNE, que gracias a estos contratos llevan a cabo actuaciones de restauración fluvial, principalmente centradas en eliminación de especies exóticas invasoras e introducción de especies de ribera autóctonas (Fundación LIMNE) en distintos tramos fluviales de la Comunidad Valenciana.

\section{EJEMPLO DE CUSTODIA DEL TERRITORIO EN LA ESPAÑA VACIADA: RECARTOGRAFÍAS}

Recartografías es una asociación cultural sin ánimo de lucro que trabaja mediante contratos de custodia, y a su vez se definen como un grupo de investigación - acción debido a los estudios que desarrollan en el territorio. Recartografías se constituye como asociación en el año 2014, tras tres años en los que sus miembros fundadores realizaron un trabajo de investigación sobre despoblación en la provincia de Teruel, con resultados como la edición de un libro, o la creación de una exposición. Fruto de esta investigación, y con la asociación registrada, sus miembros firmaron un contrato de custodia con el ayuntamiento de San Agustín, gracias al cual se les cedió durante siete años los edificios comunales de Mas Blanco, masada perteneciente a dicho municipio.

Gracias a esta cesión, Recartografías organizó distintas actividades de voluntariado mediante las cuales se rehabilitaron estos edificios, que eran la escuela, la casa de la maestra, y el horno. Su trabajo y movimiento en una aldea, en la que apenas vivía una pareja, provocó el interés de los vecinos del pueblo y de algunos propietarios de viviendas. El contacto con ellos facilitó la firma de otros contratos de custodia, esta vez con inmuebles de titularidad privada. 
Figura 1. Escuela de Mas Blanco: antes y después de su rehabilitación

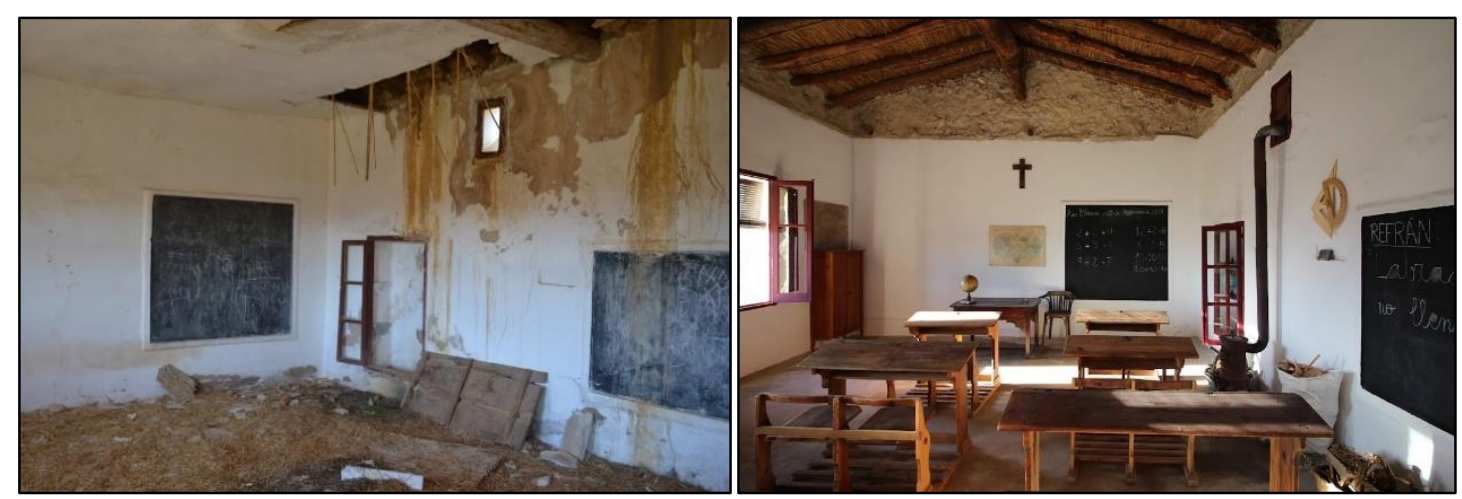

Fuente: Recartografías.

El proyecto tenía un doble objetivo: por un lado, evitar la pérdida de los edificios de la masada, destacando entre ellos la escuela; y, por otro lado, recuperar los aprovechamientos comunitarios y otras actividades que tenían lugar, como la agricultura, cada vez más minoritarias (del Romero, 2018). El trabajo de esta asociación en Mas Blanco tomó una deriva académica y divulgativa, de manera que en febrero de 2014 se celebra el primer seminario de Recartografías "Descartografía del Mundo Rural". Desde entonces, todos los meses de febrero se ha organizado un seminario, mientras que en agosto y otras épocas del año se organizaban actividades de voluntariado para rehabilitar los distintos edificios cedidos por el ayuntamiento. Desde el año 2019 estos campamentos se formalizaron como los "Cursos de Verano de la Universidad de las Masías", donde las actividades de rehabilitación de edificios se combinan con otras como talleres de carpintería y charlas formativas. Fue en este mismo año en el que se inaugura el "Museo de las Masías y la Memoria Rural", que consta de una serie de edificios rehabilitados (la escuela, la casa de la maestra y el horno; pero también el cubo, edificio en el que se elaboraba el vino, así como otros inmuebles de propiedad privada). En total, el Museo consta de siete inmuebles en los que se reparten distintas temáticas, todas relacionadas con la vida en Mas Blanco y de los masoveros, situación económica y conflictos sociales y bélicos en el siglo XX (www.recartografias.es).

Desgraciadamente, llevar a cabo un proyecto como este es muy costoso. Para rehabilitar los edificios, por ejemplo, es necesaria una inversión económica importante en materiales. Sin embargo, son pocas las ayudas destinadas a este tipo de proyectos o entidades. Hay subvenciones, sobre todo autonómicas, estatales, y cada vez más, europeas, pero para poder redactar un proyecto aspirante a estas ayudas es necesario un equipo de gente comprometida con la asociación, y este es el segundo hándicap: la falta de recursos humanos. Para este tipo de entidades es relativamente sencillo encontrar un equipo de voluntarios para una fecha concreta, pero es muy complicado mantener un grupo en el tiempo. Esta es una historia bien conocida para aquellas personas que formen parte del mundo del asociacionismo. Sin manos para trabajar, es casi imposible sacar adelante, y mantener, cualquier tipo de proyecto en una entidad sin ánimo de lucro.

Cabe destacar que el proyecto de custodia que Recartografías tiene en Mas Blanco es muy ambicioso. Por un lado, la existencia, mantenimiento, y realización de visitas guiadas al museo. Por otro lado, la organización de todo tipo de actividades, bien sean seminarios, universidades de verano o jornadas temáticas de un día de duración como son los "Findes Masoveros". Al mismo tiempo, son estas actividades las que dan sentido a la rehabilitación de estos espacios, algunos de los cuales cumplen una doble función: por un 
lado, son espacios "expositivos", formando parte del museo y representando la vida de los masoveros; y, por otro lado, son espacios "dinámicos", a los cuales se les saca un rendimiento, como sería la escuela para organizar charlas o el horno para hornear alimentos como pan o pizzas.

Figura 2. Edificios de Mas Blanco cedidos a Recartografías mediante contratos de custodia (2019)

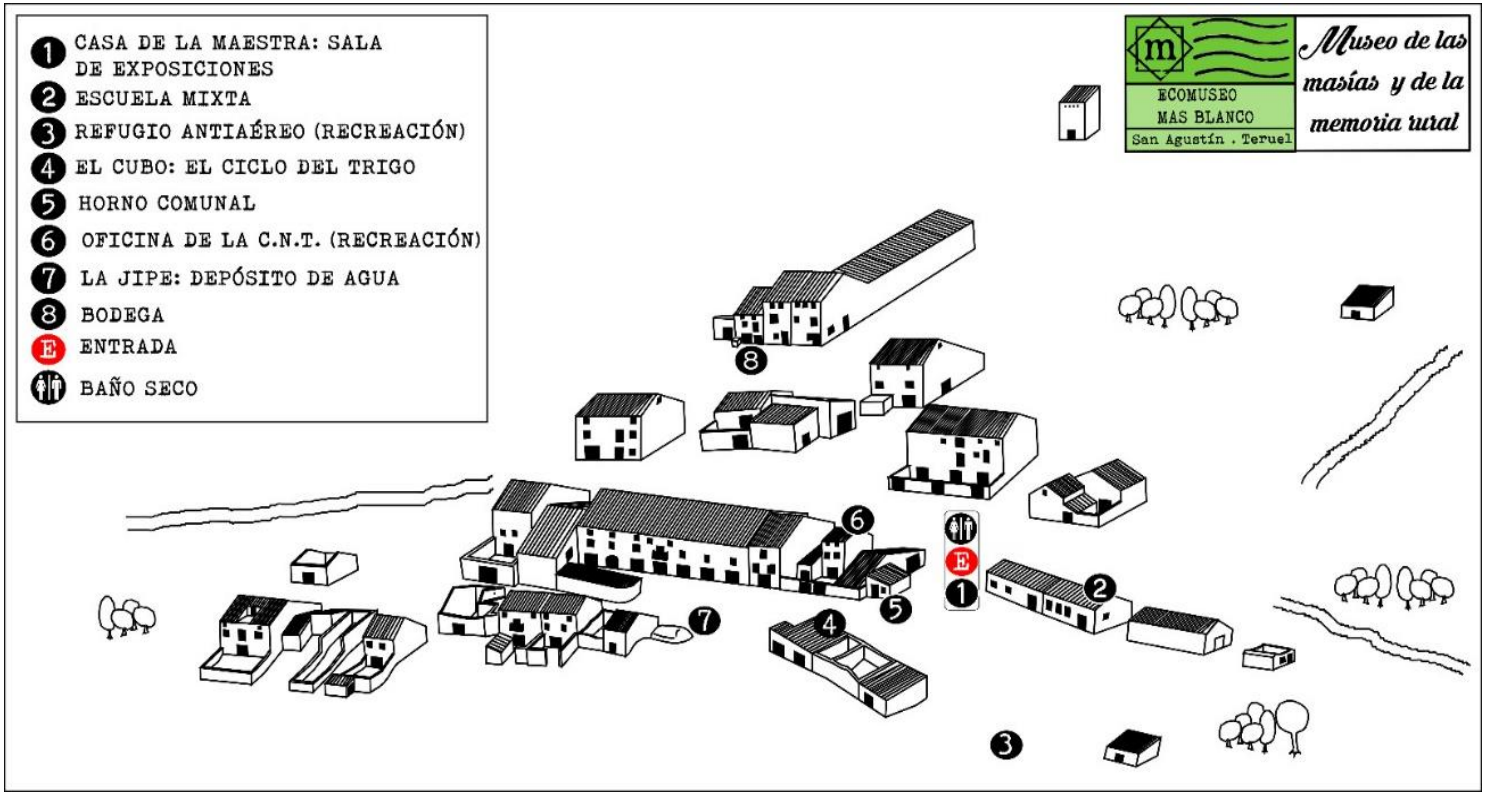

Fuente: Recartografías.

Figura 3. Seminario en la escuela de Mas Blanco y horneada en el horno comunal. Edificios rehabilitados y actividades organizadas por Recartografías

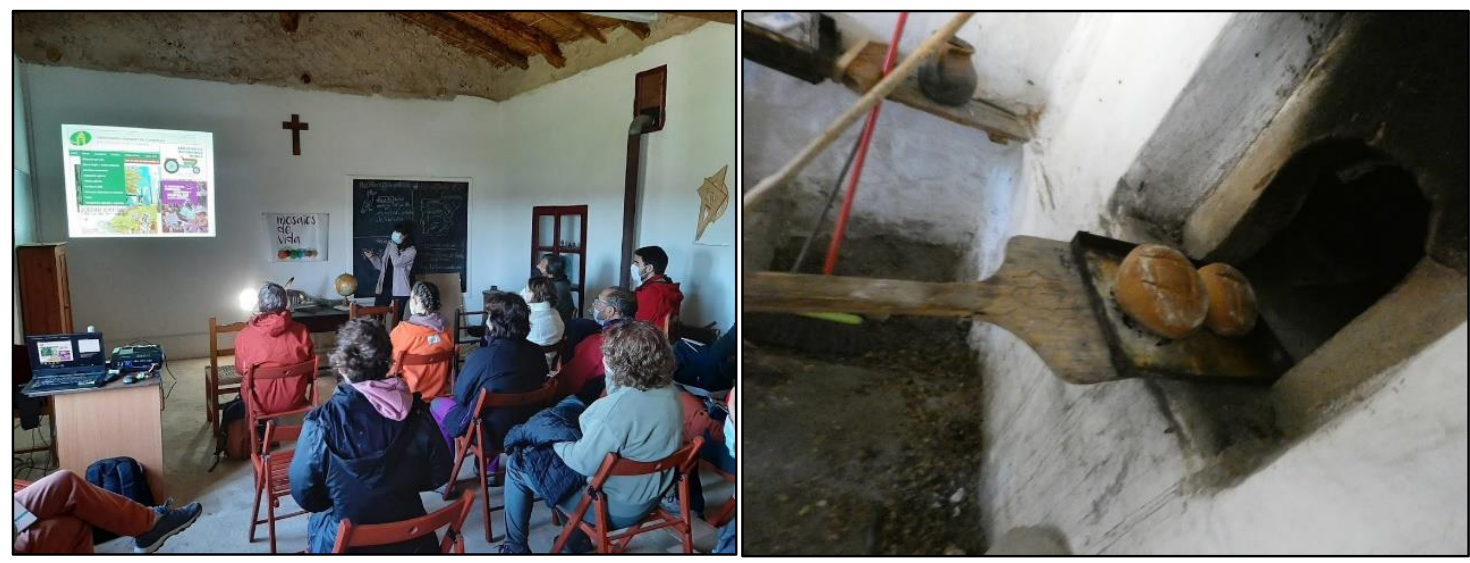

Fuente: Recartografías.

Asimismo, encontramos dificultades en el propio territorio escogido por la entidad de custodia: Mas Blanco tiene la ventaja del acceso de las dos capitales de provincia más próximas, Valencia y Teruel, puesto que se encuentra muy cerca de la "Autovía Mudéjar" (A23). Por el contrario, el acceso desde casi esta vía de comuniación consta de una pista de tierra que se resiente tras cada episodio de lluvias torrenciales, lo cual imposibilita en determinadas épocas del año poder acceder a la masada. Aunque bien es cierto que en el último año se han avanzado las labores de mantenimiento de estas pistas por parte del 
ayuntamiento de San Agustín (al cual pertenece Mas Blanco), que ha asfaltado parte de estas pistas, incluyendo los puntos más complicados de las mismas.

\section{RETOS FUTUROS TRAS OCHO AÑOS DE ACTIVIDADES}

Nos encontramos frente a un escenario de cambio global, en el cual vemos, y veremos, cómo se modifican nuestros sistemas naturales no sólo con respecto al cambio climático y sus consecuencias (aumento de fenómenos meteorológicos extremos y subida de la temperatura media entre otros), sino también con respecto a la presencia del ser humano en el territorio, y su relación con el mismo: las actividades humanas y económicas en el medio rural han pasado de la ganadería y la agricultura, a las macrogranjas y las centrales de energías renovables.

Nuestra forma de vida tiene un impacto en el territorio, en los ecosistemas, y en las especies. Nos encontramos en un contexto de mucha incertidumbre con respecto a cómo van a evolucionar estos elementos y, por otro lado, de una gran certeza: la repercusión negativa que tendrá la degradación del medio natural sobre la vida de las personas.

Esta afirmación está directamente relacionada con la teoría "One Health", definida entre otras entidades por la Organización Mundial de la Salud, como una iniciativa global que persigue mantener la buena salud de los animales y del medio ambiente, ya que repercuten directamente sobre la buena salud de las personas. Es la teoría que nos habla de la dependencia que tenemos los seres humanos de nuestro entorno natural: si este está sano, nosotros también. La pandemia del COVID-19 lo ha demostrado, como han advertido y comentado científicos como Valladares.

Ha quedado patente que las administraciones públicas no pueden llegar a todos los rincones del territorio: a veces por falta de recursos económicos, otras por falta de recursos humanos. Ahí radica la importancia de la custodia del territorio como una herramienta para poder proteger, conservar y actuar.

Por tanto, el reto principal al que se enfrenta es asumir una responsabilidad que no le corresponde legalmente, pero sí moralmente, pues es quien puede tomar el relevo de las entidades públicas. Para ello hace falta medios. Medios económicos y medios humanos. La movilización social. Tomar conciencia de la relevancia que tiene que nuestras montañas, nuestros ríos y nuestros valles gocen de buena calidad. Tomar conciencia de la importancia que tiene cultivar la tierra, no dejar que pierda sus propiedades. Y tomar conciencia de la importancia de proteger también bienes materiales, desde antiguas viviendas hasta edificios patrimoniales.

\section{CONCLUSIONES}

Queda patente que la custodia del territorio es una herramienta magnífica para llegar allí donde no llegan las administraciones públicas; para empoderar y crear unión social; y para llevar a cabo proyectos de gestión y recuperación tanto de espacios naturales como de inmuebles.

La experiencia de Recartografías nos enseña que es posible llevar a cabo proyectos de recuperación de la memoria histórica a base de constancia y compromiso. Pero cabe destacar las dificultades que estos conllevan. Es necesaria una conciencia social y un 
compromiso constante para poder realizarlos. Y, sobre todo, es importante reivindicar la necesidad de conservar estos enclaves, naturales o antrópicos, en un contexto de cambio global donde la despoblación y el cambio global amenazan con la pérdida de nuestros ecosistemas, tradiciones y memoria histórica.

\section{Referencias}

Basora, X., y Sabaté, X. (2006). Custodia del Territorio en la práctica. Manual de introducción a una nueva estrategia participativa de conservación de la naturaleza y el paisaje. Fundació Territori i paisatge. Xarxa de Custòdia del Territori. Recuperado de: https://custodiaterritorio.es/sites/default/files/recursos/custodia.practica_manual.introduccion.pdf $(15 / 12 / 2021)$.

Del Romero Renau, L. (2018). Hacia una cosmovisión renovada de lo rural: los comunes como proyecto estructurante. En L. Del Romero Renau (ed), Despoblación y abandono de la España Rural. El imposible vencido (pp. 163-164). Tirant.

Fundación LIMNE: https://www.limne.org/

Moreno, V. (2017). Custodia del Territorio: caracterización y evolución del concepto. Estudios de casos (Trabajo Final de Grado). Universitat d'Alacant. Alicante (España). Recuperado de: https://rua.ua.es/dspace/bitstream/10045/69208/1/TFG_Victor-MorenoSanchez.pdf (15/12/2021).

ONG SUBMON: https://www.submon.org/

Plataforma de Custodia del Territorio: www.custodia-territorio.es

Recartografías: www.recartografias.es

Ruiz, A., Navarro, A., y Sánchez, A. (2018). Libro blanco construyamos el futuro de la custodia del territorio. Foro de Redes y Entidades de Custodia del Territorio. Recuperado de: https://custodia-territorio.es/sites/default/files/recursos/libroblanco_cdt.pdf (15/12/2021).

Villagrasa, M. (2016). Custodia del territorio y política agraria común: oportunidades de conservación y desarrollo económico y social en el medio rural. Revista Aragonesa de Administración Pública, (148), 115-150.

Xarxa de Custòdia del Territori: https://xcn.cat/ 